\title{
Biocontrol of Rhizoctonia Stem and Root Rot of Poinsettia with Burkholderia cepacia and Binucleate Rhizoctonia
}

\author{
J. Hwang and D. M. Benson, Department of Plant Pathology, North Carolina State University, Raleigh 27695
}

\begin{abstract}
Hwang, J., and Benson, D. M. 2002. Biocontrol of Rhizoctonia stem and root rot of poinsettia with Burkholderia cepacia and binucleate Rhizoctonia. Plant Dis. 86:47-53.

Strategies for applying Burkholderia cepacia (strain 5.5B) and Pesta formulations of binucleate Rhizoctonia (BNR) isolates (BNR621 and P9023) were evaluated for biocontrol of Rhizoctonia stem and root rot of poinsettia caused by $R$. solani. During propagation, one application of $B$. cepacia suppressed stem rot, while application of either isolate of BNR did not. In contrast, after transplanting rooted poinsettias, one application of either BNR isolate was more effective for suppression of stem and root rot than application of B. cepacia. Sequential application of $B$. cepacia at propagation followed by a BNR isolate at transplanting was more effective over the crop production cycle than multiple applications of one biocontrol agent or combination application of both biocontrol agents. Root colonization by both biocontrol agents after transplanting rooted poinsettias was affected by application strategy. The least root colonization by both biocontrol agents occurred in the combination application. The highest root colonization by the BNR isolates was observed in the sequential application that provided the most effective disease control. Application of different biocontrol agents during the different production phases of poinsettia was effective for disease control, but understanding the interaction between biocontrol agents and root colonization was important to develop the best application strategy.
\end{abstract}

Poinsettia (Euphorbia pulcherrima Willd. ex Klotzsch) accounts for approximately 30\% (\$228 million) of all potted plants produced in the U.S. floriculture industry (35). In the most common production system, poinsettia cuttings are propagated in polyfoam rooting strips for 3 to 4 weeks. Rooted cuttings are transplanted to a soilless potting mix and grown for an additional 14 to 18 weeks until the typical bract color develops prior to sale (16). Stem and root rot of poinsettia caused by Rhizoctonia solani Kühn (teleomorph Thanatephorus cucumeris (Frank) Donk) is a major disease of poinsettia during both stages of plant production (29). Stem rot can occur during propagation resulting in lesions that develop on unrooted cuttings in rooting strips, which eventually girdle and kill the plants. Rooted cuttings as well as established plants are susceptible to Rhizoctonia root rot. Traditional controls for Rhizoctonia stem and root rot include sanitation and the use of fungicides (1).

Biological control of Rhizoctonia diseases has been studied extensively as an alternative to chemical control. Several

Corresponding author: D. M. Benson

E-mail: mike_benson@ncsu.edu

Research conducted by the first author in partial fulfillment of the requirements for the Ph.D. degree, North Carolina State University.

Accepted for publication 7 October 2001.

Publication no. D-2001-1119-02R

(C) 2002 The American Phytopathological Society microorganisms such as Pseudomonas spp., Trichoderma spp., and binucleate Rhizoctonia (BNR) have been identified as effective biocontrol agents $(6,24,27,30)$. One of the major constraints of biocontrol for root pathogen is that one application of a biocontrol agent may not provide seasonlong control particularly if the biocontrol agent is not rhizosphere competent (15). Maintaining an adequate population level of a biocontrol agent at the target site is essential for successful control (36).

Since the efficacy of a biocontrol agent can be affected by timing of application and type of plant growth media to which the biocontrol agent is applied (2), an application strategy to protect poinsettia plants during propagation and after transplanting is needed. In previous studies, strain 5.5B of Burkholderia cepacia controlled stem rot during propagation of poinsettia $(9,10)$, but the strain was never tested for control of root rot after transplanting. In other studies $(4,6,14,17,23)$, BNR isolates, BNR621 and P9023, controlled Rhizoctonia diseases of impatiens, snap beans, potato, and cucumber as well as Pythium pre-emergence damping-off of vinca. The objectives of this research were (i) to compare the efficacy of strain $5.5 \mathrm{~B}$ of $B$. cepacia and two isolates of BNR for control of Rhizoctonia stem and root rot in rooting strips during propagation of poinsettia and after transplanting, (ii) to evaluate different application strategies involving these biocontrol agents for disease control over both crop production stages of poinsettia, and (iii) to relate the populations of biocontrol agents on poinsettia roots and in potting mix with disease control activity.

\section{MATERIALS AND METHODS}

Poinsettia plants. Poinsettia stock plants (cv. Gutbier V-14 Glory) were maintained in 6-liter plastic pots with Metro Mix 220 (W. R. Grace Co., Cambridge, MA) on greenhouse benches. Plants were fertilized weekly with Peters 20-20-20 (NP-K, 300 ppm N) fertilizer (Scotts Co., Marysville, OH). Potassium nitrate (100 ppm N) and calcium nitrate (200 ppm N) were applied every 3 weeks, and magnesium sulfate $(2.25 \mathrm{~g} /$ liter of solution) was drenched each month. Every 3 months, a soil drench of sodium molybdate $(0.17$ $\mathrm{g} /$ liter of solution) and top-dressing of Osmocote 14-14-14 (N-P-K) (Scotts Co.) were applied. Terminal shoot cuttings approximately $10 \mathrm{~cm}$ long with two whorls of leaves were used for experiments during propagation.

Inoculum preparation. Petioles collected from poinsettia stock plants were cut in $1 \mathrm{~cm}$ lengths and autoclaved twice in glass petri dishes (60 to 70 petioles in each petri dish) lined with moistened filter paper. Four agar plugs from 5-day-old cultures of isolate RS-3 of $R$. solani from poinsettia (AG-4, deposited as NRRL 22805, USDA ARS, Peoria, IL) on potato dextrose agar (PDA, Difco Laboratories, Detroit, MI) were placed on the filter paper in the petri dishes, and incubated at approximately $25^{\circ} \mathrm{C}$ for 7 days prior to use.

Biocontrol agents. Cultures of $\mathrm{B}$. $\mathrm{ce}$ pacia (strain 5.5B) were initiated by streaking cells from a stock cell suspension in deionized water, stored at $4^{\circ} \mathrm{C}$, on PDA and incubating the cultures at approximately $25^{\circ} \mathrm{C}$ for 3 days. A single colony from the fresh culture on PDA was transferred in nutrient broth medium (Difco Laboratories) and incubated at approximately $25^{\circ} \mathrm{C}$ on a rotary shaker at $200 \mathrm{rpm}$ for 5 days. Cells were collected by centrifuging cultures at $3,500 \times g$ for $20 \mathrm{~min}$. Cells were resuspended in deionized water at $10^{9} \mathrm{CFU} / \mathrm{ml}$ by use of a spectrophotometer at $530 \mathrm{~nm}$ (Spectronic 20D, Milton Roy, Rochester, NY).

Binucleate Rhizoctonia (BNR) isolates, BNR621 (originally GC-232) and P9023, isolated from pine bark in a pot growing an azalea and the roots of plantain, respectively, were from the collection of $\mathrm{E}$. Echandi, North Carolina State University (17). Three, 7-mm-diameter agar disks of an isolate from the margin of actively growing cultures on PDA were transferred 
to a 1-liter flask containing $50 \mathrm{~g}$ of twice autoclaved wheat bran and $50 \mathrm{ml}$ of deionized water. The cultures were grown for 5 days on the lab bench at approximately $25^{\circ} \mathrm{C}$ then formulated separately into Pesta granules as described by Connick et al. (12). The resulting formulation contained $20 \%$ biomass of a given BNR isolate.

Biocontrol experiments. Three types of experiments were conducted in the greenhouse. In the first experiment, a single application of a putative biocontrol agent was made at the start of the propagation cycle. A $180-\mathrm{ml}$ cell suspension of B. cepacia as described above, was applied to a dry wedge-shaped rooting strip (OASIS Wedge Growing Medium, Smithers-Oasis, Kent, $\mathrm{OH})$. The 10-unit foam strip with molded plastic support was cut in half so that there were five units per strip, and a strip was considered to be one replicate. The five-unit strip completely soaked up the suspension $(36 \mathrm{ml}$ per individual wedge-shaped unit). For application of BNR isolates at propagation, $0.22 \mathrm{~g}$ of Pesta granules of BNR621 or P9023 were top-dressed to the surface of each prewetted rooting unit (total $1.1 \mathrm{~g}$ per fiveunit strip and equivalent to a rate of $0.6 \%$ on a granule volume to wedge-shaped unit volume basis). Untreated strips were moistened with water as a control. Five poinsettia cuttings from stock plants were placed in preformed holes of each strip. A petiole segment colonized by $R$. solani was placed on the long-axis surface of each rooting strip $2 \mathrm{~cm}$ away from each cutting. Strips with cuttings were arranged on greenhouse bench with a misting system that cycled on $1 \mathrm{~min}$ per h, 14 times a day in a randomized complete block design with three replications.

Stems of each cutting in the strip were examined weekly for 3 weeks for extent of stem rot. Stem rot severity was rated based on the following scale: $1=$ no disease, $2 \leq$ $25 \%$ of the stem with lesions of $R$. solani, $3=25$ to $50 \%$ of the stem with lesions, $4 \geq$ $50 \%$ of the stem girdled, and $5=$ stem completely girdled or cutting collapsed. Disease severity of the five cuttings in a strip was averaged and represented as one replicate. Data were analyzed with PROC GLM (SAS Institute, Inc., Cary, NC) with treatment as a fixed effect in a one-way analysis of variance (ANOVA). Significant differences among means for disease severity at each sampling period were determined with Duncan's multiple range test $(P=0.05)$. The experiment was performed three times and means were pooled across experiments for analysis since variances were homogenous.

In the second type of experiment, a single application of the biocontrol agent was made at transplanting of 3- to 4-week-old healthy rooted cuttings to 1.6-liter plastic pots containing a peat moss based potting mix (Fafard Mix No. 4, Fafard, Inc., Anderson, SC). The mix had a $\mathrm{pH}$ of 5.5 to
6.5 after wetting. A $160-\mathrm{ml}$ cell suspension of $B$. cepacia as described above (1\% volume of biocontrol agent per volume of growth medium) was drenched onto the potting mix in a pot after transplanting.

For application of BNR isolates, the potting mix was adjusted to approximately $65 \%$ moisture content then amended with $4.5 \mathrm{~g}$ of Pesta granules of a BNR isolate per liter of potting mix $(0.6 \%$ on a Pesta volume to potting mix volume basis) by rotating granules and potting mix in a partially inflated plastic bag until thoroughly mixed. Amended mix was stored in the bags under shade in the greenhouse where daily temperatures ranged from 18 to $30^{\circ} \mathrm{C}$ for 1 day prior to transplanting healthy rooted poinsettia plants to allow colonization of the mix by BNR isolates. A third set of transplanted rooted cuttings was left untreated as an inoculated control. Enough rooted cuttings were established for each treatment so that three replicates could be examined for stem and root rot at each sampling date as described below. Transplanted rooted cuttings were inoculated by burying six petiole segments colonized by $R$. solani $1 \mathrm{~cm}$ below the surface of the potting mix, $3 \mathrm{~cm}$ from the stem of a plant. Transplants were fertilized the same as stock plants described above. Severity of stem rot as described above and root rot was recorded weekly for 4 weeks after inoculation. Root rot was rated based on the following scale: $1=$ no disease, $2 \leq$ $10 \%$ of the root system discolored, $3=11$ to $25 \%$ of the root system discolored, $4=$ 26 to $50 \%$ discolored, and $5 \geq 50 \%$ discolored. Stem and root rot was assessed for each plant by removing the potting mix to examine the stems and roots. Stem and root rot severity at each sampling period was averaged. There were three replications for each biocontrol agent and untreated control arranged in a randomized complete block on the greenhouse bench. The experiment was performed three times and means were pooled across experiments for analysis of variance since variances were homogenous. Significant differences among means for disease severity at each sampling period were determined with Duncan's multiple range test $(P=0.05)$.

In the third experiment, three different application strategies were tested on cuttings as they were rooted during the propagation cycle then continued at transplanting with only the rooted cuttings that remained healthy.

For sequential application, B. cepacia was applied to rooting strips to protect poinsettia cuttings during propagation as described above. After 3 to 4 weeks, healthy rooted cuttings without stem or root lesions caused by $R$. solani were selected and transplanted to potting mix amended with the Pesta formulation of either isolate of BNR as described above. Cuttings with apparent lesions were discarded at transplanting. In the multiple application strategy, rooting strips were treated with $B$. cepacia with an additional drench of B. cepacia applied to the potting mix as described above after transplanting of healthy poinsettia plants protected by $B$. cepacia during root propagation. For the combination application strategy, both $B$. cepacia and the Pesta formulation of either BNR isolate were applied to rooting strips, followed by a second application of both biocontrol agents to the potting mix at transplanting of healthy poinsettia cuttings. Cuttings infected with $R$. solani during propagation were discarded at transplanting. Data collected from the treatments of each BNR isolate were combined since no differences over BNR isolates were observed.

In all application strategies, poinsettia plants were inoculated twice with petiole inoculum of $R$. solani, once after application of biocontrol agents to rooting strips and then after transplanting rooted poinsettia cuttings. Since cuttings without biocontrol agent application (inoculated control) were severely infected during propagation, noninoculated rooted cuttings were transplanted to serve as an inoculated control for this phase of production. Poinsettia plants were examined weekly for stem and root rot severity for 52 days as described above, beginning when cuttings were placed in strips and continuing for 21 days after transplanting. Severity of stem and root rot from each sampling period was averaged. There were three replications for each application strategy and an inoculated control arranged in a randomized complete block on the greenhouse bench. The experiment was performed three times. Significant differences among means for disease severity at each sampling period were determined with Duncan's multiple range test $(P=0.05)$. Means were pooled across experiments for ANOVA since variances were homogenous.

Population assay of biocontrol agents. The population dynamics of B. cepacia and BNR isolates on poinsettia roots and in potting mix were determined when the biocontrol agents were applied after transplanting. In a previous study, Cartwright and Benson (8) found that the population of strain $5.5 \mathrm{~B}$ of $B$. cepacia declined dramatically from $\log 9.1 \mathrm{CFU} / \mathrm{ml}$ to $\log 7.5$ $\mathrm{CFU} / \mathrm{ml} 3$ weeks after soaking rooting strips with the bacterium, so this phase of population dynamics was not included in the present study. Healthy noninoculated rooted cuttings approximately 3 to 4 weeks old were transplanted to Farfard Mix No. 4 in 1.6 liter pots. Potting mix was amended with the Pesta formulation of a BNR isolate or drenched with a suspension of $B$. cepacia after transplanting as described above. Noninoculated cuttings were propagated in rooting strips treated with either $B$. cepacia (sequential and multiple application) or B. cepacia and a BNR isolate (combination application). Rooted cuttings 
were transplanted to the potting mix amended with $B$. cepacia (multiple application), a BNR isolate (sequential application), or B. cepacia and a BNR isolate (combination application). Enough plants were treated so two replicates of each treatment could be sampled destructively at five times over a 9-week period after transplanting.

Poinsettia plants were removed from the potting mix at $1,3,5,7$, and 9 weeks after application of biocontrol agents, and adhering mix was gently washed off the roots under running tap water. Washed root tissue was blot-dried, and $1 \mathrm{~g}$ of root pieces per plant were dissected 0.5 to $1 \mathrm{~cm}$ long. Root pieces were divided and plated on five petri dishes of alkaline water agar with antibiotics (18) to recover BNR isolates. After 3 days, each developing colony on alkaline water agar was assumed to result from a single propagule on the root. For enumeration of $B$. cepacia, $1 \mathrm{~g}$ of root pieces per plant were vortexed vigorously in sterile water for $2 \mathrm{~min}$, then $0.1-\mathrm{ml}$ aliquots were plated on trypan bluetetracycline (TB-T) (19) after a series of dilutions. After 5 days, root colonization of the biocontrol agent was assayed by counting the number of colonies on the selective media.

A multiple-pellet soil-sampler (MPSS) described by Henis et al. (20) was used for population assay of BNR isolates in the potting mix samples. Approximately $30 \mathrm{~g}$ of potting mix from a pot was placed in a petri dish, then pellets were acquired by tapping the MPSS gently into the potting mix. Three sets of sub-samples were collected in this manner from each of two pots per treatment. The pellets were transferred to alkaline water agar medium and incubated for 3 days at approximately $25^{\circ} \mathrm{C}$. The number of pellets from which BNR grew was observed under a dissecting microscope. The first order of the Poisson distribution was applied to estimate number of propagules of BNR isolates per gram of potting mix from the number of pellets colonized by BNR (20).

Potting mix was assayed for $B$. cepacia by stirring $10 \mathrm{~g}$ of potting mix from each pot in $30 \mathrm{ml}$ distilled water for $20 \mathrm{~min}$. Aliquots were clarified through filter paper (Fisherbrand filter paper P8, Fisher Scientific, Pittsburgh, PA). A dilution series of collected aliquots was spread at $0.1 \mathrm{ml}$ per plate to TB-T. There were three plates per 10 -g sample and two pots were assayed per treatment. The plates were incubated at approximately $25^{\circ} \mathrm{C}$ for 5 days and CFU of B. cepacia were counted. Bacterial populations on roots and in potting mix were transformed as the logarithms (base 10) of CFU per gram of root and per gram of potting mix prior to analysis (32). The experiments were conducted twice and the data were pooled based on homogeneity of variances. Significant differences among means from population assay of biocontrol agents at each sampling period were determined with Duncan's multiple range test $(P=0.05)$.

\section{RESULTS}

Efficacy of biocontrol agents during propagation. A single application of $B$. cepacia to rooting strips at the time of propagating poinsettia cuttings suppressed Rhizoctonia stem rot. At each sampling date, after soaking rooting strips with $B$. cepacia, diseases severity was less than for the inoculated control (Fig. 1). Severity of stem rot was 2.1 at 24 days after inoculation compared to a severity of 4.3 in the inoculated control (Fig. 1). Stem rot severity for cuttings in strips treated with the Pesta formulation of either isolate of binucleate Rhizoctonia sp. was not different from the inoculated control $(P=0.05)$ at any sampling date (Fig. 1).

Efficacy of biocontrol agents after transplanting. The three biocontrol agents tested suppressed stem and root rot when they were applied to potting mix. When the Pesta formulation of the BNR isolates was mixed into the potting mix at transplanting, Rhizoctonia stem and root rot was less $(P$ $=0.05$ ) at each sampling date regardless of isolate tested compared to the inoculated control (Fig. 2). Disease severity of poinsettias in pots treated with BNR621 and P9023 at 28 days after inoculation was 1.5 and 1.6 , respectively, compared to 4.3 in the inoculated control (Fig. 2). Although significantly less $(P=0.05)$, stem and root rot developed on poinsettia plants in the mix drenched with B. cepacia compared to the inoculated control, disease severity was 3.0 at 28 days after inoculation and plants

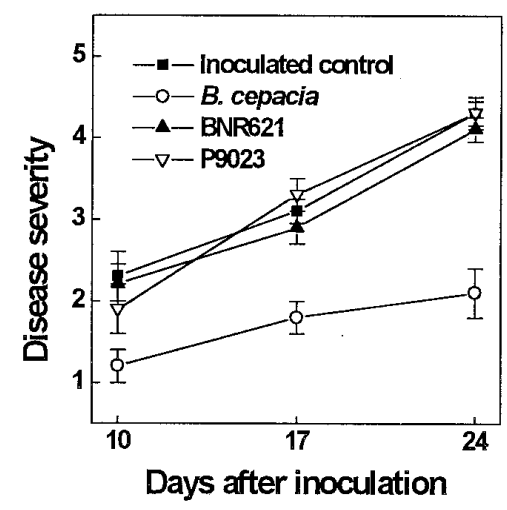

Fig. 1. Disease severity of Rhizoctonia stem rot on poinsettia cuttings caused by Rhizoctonia solani during root propagation where rooting strips were treated with either strain $5.5 \mathrm{~B}$ of Burkholderia cepacia or a Pesta formulation of binucleate Rhizoctonia isolates BNR621 or P9023 compared to the inoculated control. Disease severity is based on 1 to 5 scale where $1=$ no disease and $5=$ stem completely girdled and cutting collapsed as described in Materials and Methods. Values plotted are means of three replications per experiment pooled over three experiments. Bars represent standard error of the mean. were not commercially acceptable (Fig. 2). The BNR isolates were not pathogenic to poinsettias in the absence of $R$. solani (data not shown).

Efficacy of biocontrol agents during multiple, combination, or sequential applications. When multiple applications of $B$. cepacia were made at propagation and additionally after transplanting, disease severity of stem rot at the end of the propagation cycle was 1.9 compared to 4.2 in the inoculated control (Fig. 3). During the next 28 days following transplanting selected rooted cuttings, inoculation, and drenching with an additional application of $B$. cepacia to the potting mix, disease severity rose from 1.4 to 2.7 at 52 days after propagation, which was less $(P=0.05)$ than the 4.0 for poinsettia in the inoculated control (Fig. 3).

When a combination of both BNR and B. cepacia was applied to the rooting strips at propagation, disease severity of stem rot at the end of the propagation cycle was 3.2 , and greater than the rating of $2.0(P=$ $0.05)$ for poinsettia cuttings in the rooting strips treated with B. cepacia alone (Fig. 3). After transplanting healthy cuttings and inoculating, disease severity of poinsettia in BNR and B. cepacia treated potting mix 52 days after propagation of cuttings was 3.0, which was less $(P=0.05)$ than the 4.0 for poinsettia in the inoculated control (Fig. 3).

Sequential application of B. cepacia to the rooting strips, followed by incorporation of the Pesta formulation of the BNR

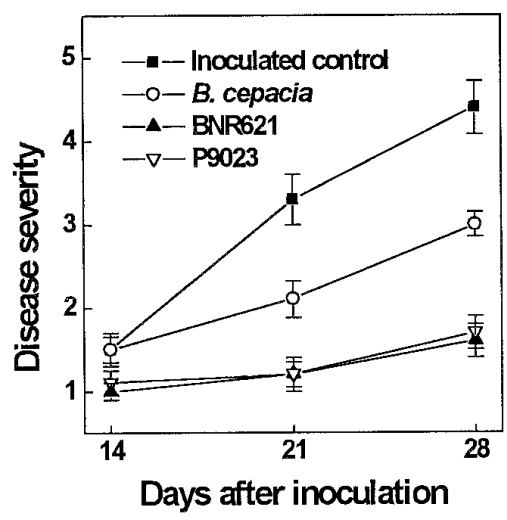

Fig. 2. Disease severity of Rhizoctonia stem and root rot on poinsettia plants after transplanting in potting mix inoculated with petiole segments colonized by Rhizoctonia solani and amended with either Burkholderia cepacia or a Pesta formulation of binucleate Rhizoctonia isolates (BNR621 and P9023) compared to the inoculated control. Disease severity is the mean of stem rot rating of 1 to 5 scale where $1=$ no diseases and $5=$ stem completely girdled and cutting collapsed, and a root rot rating of 1 to 5 scale where $1=$ no disease and $5 \geq 50 \%$ of roots discolored as described in Materials and Methods. Values plotted are means of three replications per experiment pooled over three experiments. Bars represent standard error of the mean. 
isolates at transplanting gave the highest level of disease control among the application strategies tested $(P=0.0031)$. When $B$. cepacia was applied at the rooting strip stage, disease severity averaged 2.0 after 24 days of propagation, while disease severity was 2.1 for transplanted and inoculated poinsettia in BNR treated pots 52 days after propagation compared to 4.0 for the inoculated control (Fig. 3).

Population dynamics of biocontrol agents. One week after B. cepacia was applied to the surface of potting mix containing rooted poinsettia plants, the population on the poinsettia root system was log 7.01 CFU/g root compared to $\log 6.03$ CFU/g in the potting mix (Fig. 4). Colonization of poinsettia roots by $B$. cepacia remained near $\log 7 \mathrm{CFU} / \mathrm{g}$ root for up to 5 weeks after initial application, then dropped to about $\log 5 \mathrm{CFU} / \mathrm{g}$ root after 9 weeks. Conversely, in the potting mix, the population of $B$. cepacia declined rapidly and reached a nondetectable level 9 weeks after application (Fig. 4).

Isolates of BNR colonized the poinsettia root system when they were introduced to the potting mix at transplanting. The population of isolates BNR621 and P9023 in the poinsettia root system reached 134 and 142 propagules per gram root, respectively, at 1 week after application, then declined gradually to 52 and 46 propagulesper gram root 9 weeks after treatment (Fig. 5). Populations of BNR isolates in the potting mix ranged from 8 to 17 propagules per gram mix over the 9week period after application (Fig. 5). No difference between isolates BNR621 and P9023 was found $(P=0.05)$ in root popu- lations or potting mix populations at any given sampling period.

Effect of application strategy on population dynamics of biocontrol agents. Root colonization by the biocontrol agents after transplanting of poinsettia varied depending on the application method tested. When multiple applications of $B$. cepacia were made to the rooting strips as well as to the potting mix at transplanting, root colonization of $B$. cepacia was $\log 7.70 \mathrm{CFU} / \mathrm{g}$ root 1 week after transplanting then dropped to less than log $5 \mathrm{CFU} / \mathrm{g}$ root after 9 weeks (Fig. 6). For the combination application, where both BNR and B. cepacia were applied at propagation and at transplanting, initial root population of $B$. cepacia was $\log 7$ $\mathrm{CFU} / \mathrm{g}$ root at 1 week after transplanting

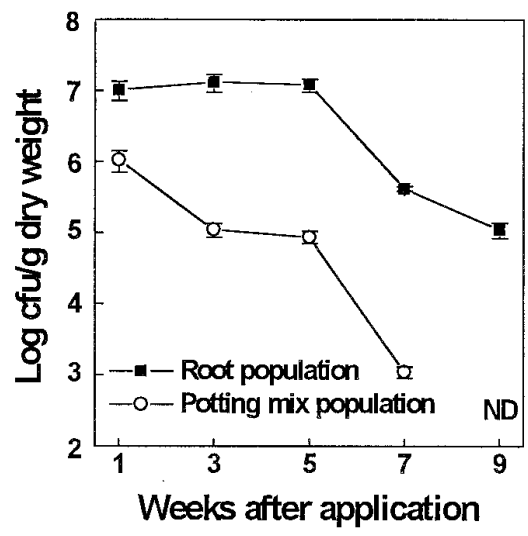

Fig. 4. Population dynamics of strain 5.5B of Burkholderia cepacia on poinsettia roots and in the potting mix after the mix was drenched with $B$. cepacia at transplanting rooted poinsettia cuttings. $\mathrm{ND}=$ nondetectable. Values plotted are means of two replications per experiment pooled over three experiments. Bars represent standard error of the mean.

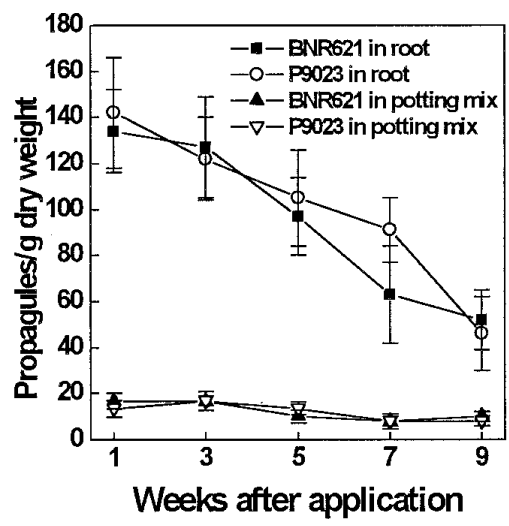

Fig. 5. Population dynamics of binucleate Rhizoctonia (BNR) isolates (BNR621 and P9023) on poinsettia roots and in the potting mix after a Pesta formulation of each BNR isolate was introduced in the mix used to transplant rooted poinsettia cuttings. Values plotted are means of two replications per treatment pooled over three experiments. Bars represent standard error of the mean. 
but declined sharply to only $\log 3.88$ $\mathrm{CFU} / \mathrm{g}$ root after 9 weeks. In the sequential application with B. cepacia at propagation followed by BNR amendment at transplanting, the population of B. cepacia in the poinsettia roots was lower than in multiple applications of $B$. cepacia. Root population in the sequential application 1 week after transplanting was log 7.66 $\mathrm{CFU} / \mathrm{g}$ root and decreased to $\log 4.32$ $\mathrm{CFU} / \mathrm{g}$ root during the 9 -week period (Fig. 6).

When isolates BNR621 and P9023 were amended to the potting mix in combination with B. cepacia, root colonization of BNR isolates was less $(P=0.05)$ than in sequential application at any sampling date. Root colonization after 1 week was only 69 and 71 propagules per gram root for isolate BNR621 and isolate P9023, respectively. Over time, root colonization by the BNR isolates declined to 20 and 23 propagules per gram root for isolates BNR621 and P9023 at 9 weeks after application, respectively (Fig. 7). When either BNR isolate was applied to the potting mix sequentially after B. cepacia was applied to the rooting strips, subsequent root colonization ranged from 103 to 112 propagules per gram root 1 week after BNR application (Fig. 7). A gradual decline in root colonization occurred over the next 8 weeks resulting in 42 to 51 propagules per gram root by week 9. No difference in root colonization $(P=$ $0.05)$ was found between the two BNR isolates.

\section{DISCUSSION}

Timing of application, application strategy, and establishment of biocontrol agents at the target area are the critical elements for successful biocontrol $(2,5,36)$. In this study, two different biocontrol agents (BNR fungi and B. cepacia), previously shown to have biocontrol activity against Rhizoctonia diseases $(9,10,14,25)$ were applied and evaluated at two distinct production stages of poinsettia. Effective control of Rhizoctonia stem and root rot was greatly affected by the timing of application of each biocontrol agent. At propagation, only B. cepacia was effective in control of Rhizoctonia stem rot of poinsettia. Several studies have demonstrated that antibiosis is a major factor in biocontrol of plant diseases by B. cepacia $(11,22,26,28)$. Polyfoam rooting strips have high water holding capacity and an initial low indigenous population of bacteria. This is an ideal environment for $B$. cepacia where antibiotics such as pyrrolnitrin can diffuse throughout the rooting strips to inhibit $R$. solani (8).

BNR isolates, BNR621 and P9023, showed no biocontrol activity of Rhizoctonia stem rot when they were applied at propagation to rooting strips. On the contrary, both BNR isolates showed more suppression of Rhizoctonia stem and root rot than B. cepacia when the Pesta formu- lation was amended to the potting mix at transplanting. Postulated mechanisms of biocontrol of Rhizoctonia diseases by binucleate Rhizoctonia sp. include induction of systemic host resistance and competition for invasion sites or nutrients $(7,21,25,34,37)$. When BNR isolates were introduced to rooting strips, stems of poinsettia cuttings had relatively little surface area for colonization and BNR fungi are not thought to produce antibiotics active against $R$. solani. However, BNR isolates could colonize poinsettia roots to induce systemic host resistance after transplant. Poromarto et al. (34) demonstrated that precolonization of root tissue by binucleate Rhizoctonia was critical to induce host resistance for inhibition of $R$. solani on soybean.

Multiple applications of the same biocontrol agent, integrated use of chemicals with biocontol agents, or combined application of two or more biocontrol agents may be needed to achieve season-long control of plant diseases $(13,14,38)$. Our results demonstrated that sequential application where rooting strips were treated with B. cepacia, followed by application of
BNR isolates at transplanting rooted poinsettia cuttings, was the most effective strategy for control of stem and root rot compared to the combination or multiple application of these two biocontrol agents. Even though previous studies indicated that mixtures of biocontrol agents acted synergistically in disease control $(31,38)$, effects were not additive where $B$. cepacia and BNR isolates were combined in this study. An antagonistic interaction may be involved between $B$. cepacia and the BNR fungi. Since binucleate Rhizoctonia and $R$. solani are closely related, antibiotics produced by $B$. cepacia may interfere with biocontrol activity by BNR isolates. Both BNR isolates and B. cepacia colonize root tissue of poinsettia cuttings, therefore, competition for space between these two biocontrol agents also can be speculated.

Many studies have shown that colonization of biocontrol agents at the target site is a prerequisite for suppression of plant pathogens $(3,33,36)$. Population dynamics of B. cepacia and BNR isolates in this study demonstrated that colonization of poinsettia roots by these biocontrol agents is closely related to biocontrol activity and

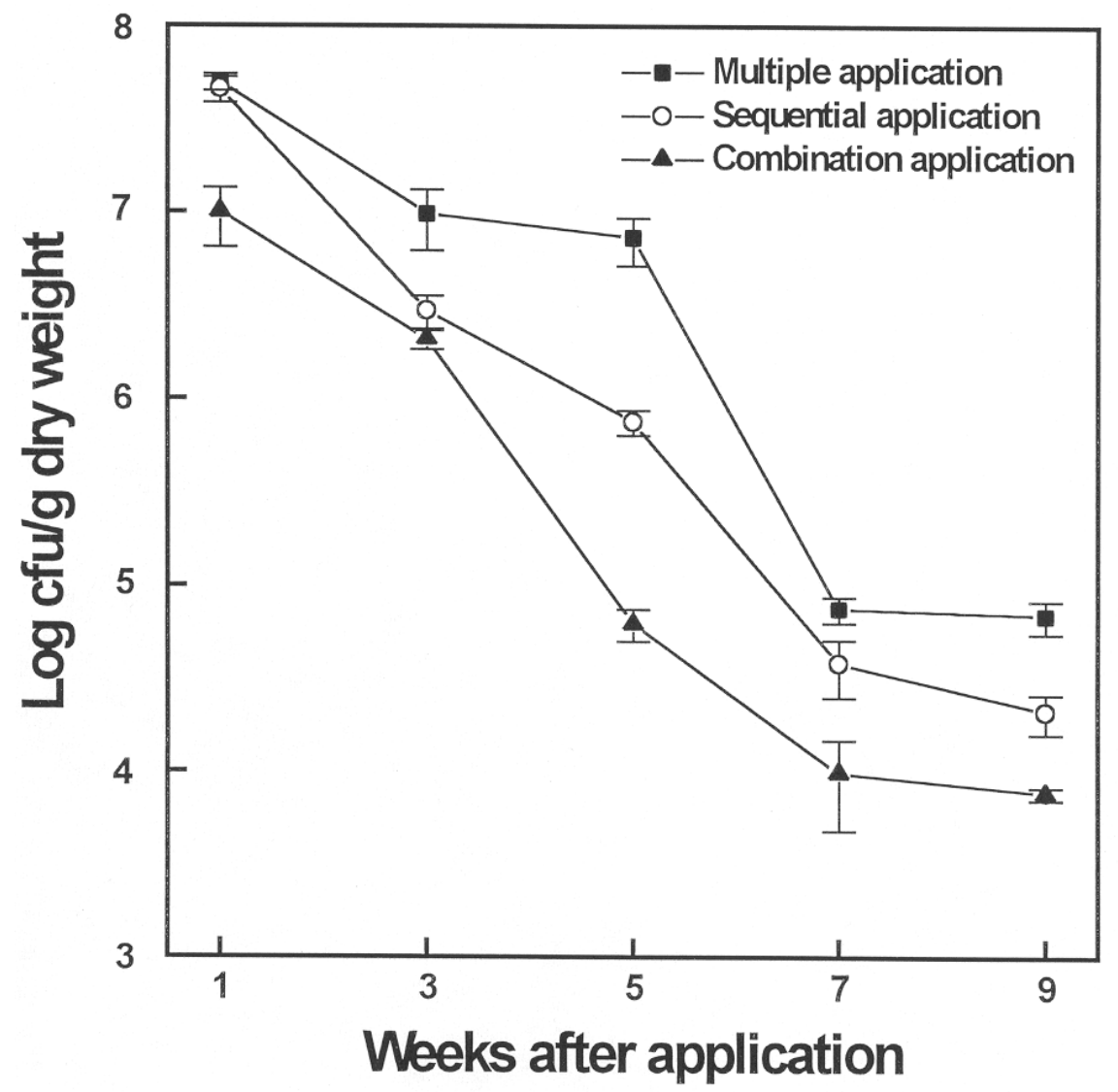

Fig. 6. Effect of application strategy on root colonization by strain 5.5B of Burkholderia cepacia on poinsettia after transplanting rooted poinsettia cuttings. In the multiple application, cuttings were treated in rooting strips with $B$. cepacia and transplanted to potting mix drenched with $B$. cepacia. In the sequential application, cuttings were treated in rooting strips with B. cepacia and transplanted to potting mix amended with binucleate Rhizoctonia (BNR) isolates. In the combination application, cuttings were treated in rooting strips with both $B$. cepacia and BNR isolates and transplanted to potting mix amended with both $B$. cepacia and BNR isolates. Values are means of two replications per experiment pooled over two experiments. Bars represent standard error of the mean. 
affected by application strategy. Both $B$. cepacia and BNR isolates were good colonizers of poinsettia roots and maintained the initial high population level up to 5 weeks after application, whereas, the population of these biocontrol agents was much lower and declined more rapidly in the potting mix. Even though the population of $B$. cepacia on poinsettia roots was greatest with the multiple application of $B$. cepacia applied to rooting strips followed by a second application at transplanting, the most effective biocontrol of stem and root rot was achieved in sequential application where B. cepacia was applied to rooting strips followed by amendment of potting mix with a Pesta formulation of BNR isolates at transplanting. Our data suggest that BNR isolates played a more important role than B. cepacia in biocontrol of stem and root rot control after transplanting. Root colonization of poinsettia by BNR isolates when applied sequentially was consistently higher than when applied in combination with $B$. cepacia. Disease suppression by the BNR isolates in sequential application also was consistently higher than in the combination application. Considering that more biocontrol activity was observed when greater colonization of poinsettia roots by the BNR isolates occurred, we conclude that root colonization by these BNR isolates was critical for biocontrol of Rhizoctonia stem and root rot of poinsettia.

Our findings indicate that sequence of biocontrol agent application was critical for control of stem and root rot. The mode of action of B. cepacia and BNR isolates was important in determining the phase of poinsettia production in which these biocontrol agents provided the best control. Finally, improving the efficacy of these biocontrol agents may be achieved by providing supplemental nutrients to improve colonization or additional applications of the biocontrol agent to maintain populations at a high level for a longer period since disease control activity was closely related to root colonization of these two biocontrol agents.

\section{ACKNOWLEDGMENTS}

This research was supported in part by the North Carolina Agricultural Research Service, North Carolina State University, Raleigh, and by a grant from the American Floral Endowment, Glen Carbon, IL. The authors thank K. C. Parker for technical assistance.

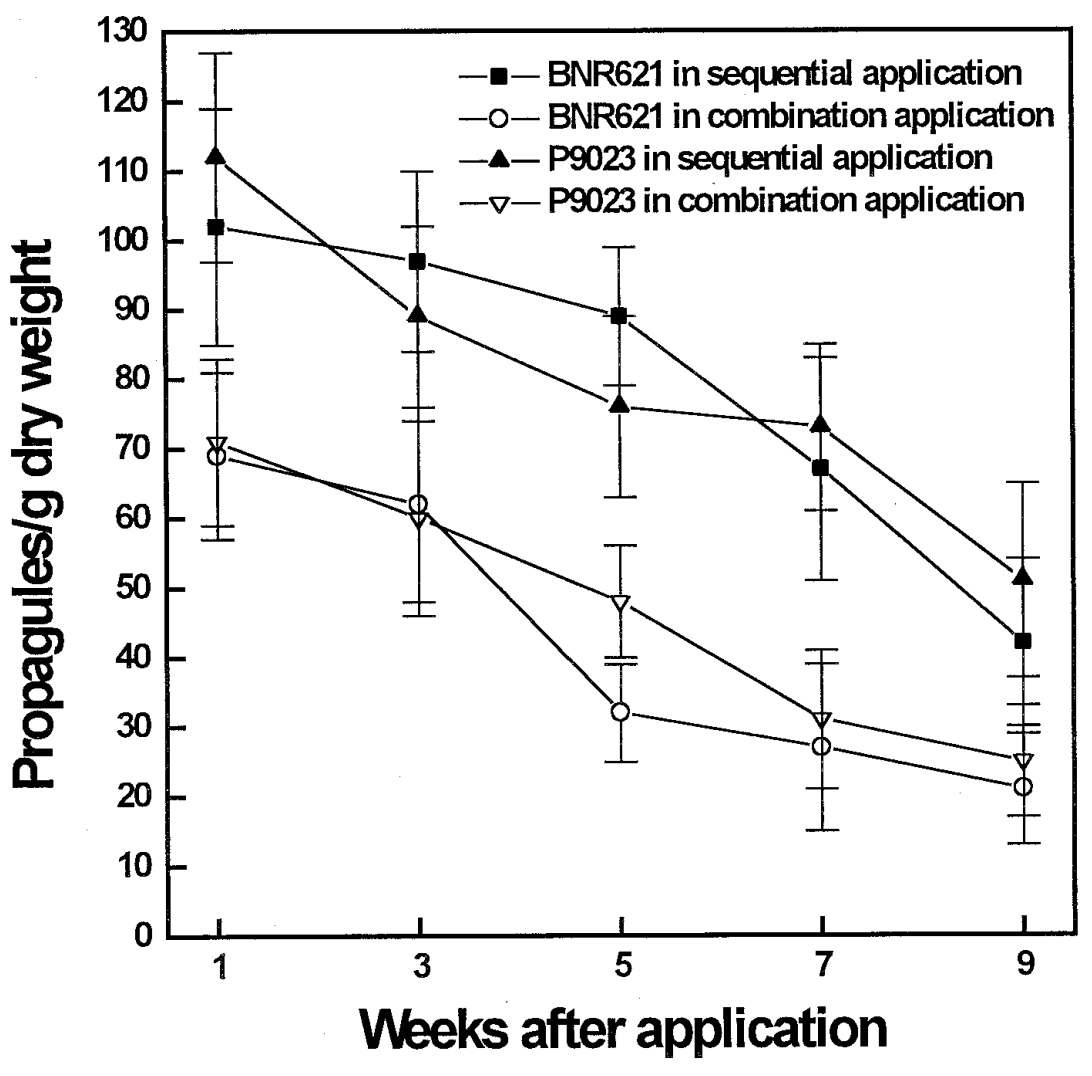

Fig. 7. Effect of application strategy on poinsettia root colonization by binucleate Rhizoctonia (BNR) isolates BNR621 and P9023 in a Pesta formulation after transplanting rooted poinsettia cuttings. In the sequential application, cuttings were treated in rooting strips with B. cepacia and transplanted to potting mix amended with BNR isolates. In the combination application, cuttings were treated in rooting strips with both $B$. cepacia and BNR isolates and transplanted to potting mix amended with both $B$. cepacia and BNR isolates. Values are means of two replications per experiment pooled over two experiments. Bars represent standard error of the mean.
LITERATURE CITED

1. Bailey, D. A. 1990. Cultural practices and IPM for Poinsettias. N C Flower Growers' Bull. 35:2-6.

2. Baker, K. F., and Cook, R. J. 1974. Biological control in plant pathology. Pages 50-55 in: Biological Control of Plant Pathogens. K. F. Baker, and R. J. Cook, eds. W. H. Freeman and Company, San Francisco.

3. Bull, C. T., Weller, D. M., and Thomashow, L. S. 1991. Relationship between root colonization and suppression of Gaeumannomyces graminis var. tritici by Pseudomonas fluorescens strain 2-79. Phytopathology 81:954-959.

4. Burns, J. L., and Benson, D. M. 2000. Biocontrol of damping-off of Catharanthus roseus caused by Pythium ultimum with Trichoderma virens and binucleate Rhizoctonia fungi. Plant Dis. 84:644-648.

5. Campbell, R. 1989. Biological Control of Microbial Plant Pathogens. Cambridge University Press, Cambridge.

6. Cardoso, J. E., and Echandi, E. 1987. Biological control of Rhizoctonia root rot of snap bean with binucleate Rhizoctonia-like fungi. Plant Dis. 71:167-170.

7. Cardoso, J. E., and Echandi, E. 1987. Nature of protection of bean seedlings from Rhizoctonia root rot by binucleate Rhizoctonia-like fungus. Phytopathology 77:1548-1551.

8. Cartwright, D. K., and Benson, D. M. 1994. Effect of population dynamics of Pseudomonas cepacia and Paecilomyces lilacinus on colonization of polyfoam rooting cubes by Rhizoctonia solani. Appl. Environ. Microbiol. 60:2852-2857.

9. Cartwright, D. K., and Benson, D. M. 1995. Comparison of Pseudomonas species and application techniques for biocontrol of Rhizoctonia stem rot of poinsettia. Plant Dis. 79:309313.

10. Cartwright, D. K., and Benson, D. M. 1995. Optimization of biological control of Rhizoctonia stem rot of poinsettia by Paecilomyces lillacinus and Pseudomonas cepacia. Plant Dis. 79:301-308.

11. Cartwright, D. K., Chilton, W. S., and Benson, D. M. 1995. Pyrrolnitrin and phenazine production by Pseudomonas cepacia, strain 5.5B a biocontrol agent of Rhizoctonia solani. Appl. Microbiol. Biotechnol. 43:211-216.

12. Connick, W. J., Boyette, C. D., and McAlpine, J. R. 1991. Formulation of mycoherbicides using a pasta-like process. Biol. Control 1:281-287.

13. Conway, K. E., Maness, N. E., and Motes, J. E. 1997. Integration of biological and chemical controls for Rhizoctonia aerial blight and root rot of rosemary. Plant Dis. 81:795-798.

14. Cubeta, M. A., and Echandi, E. 1991. Biological control of Rhizoctonia and Pythium damping-off of cucumber: an integrated approach. Biol. Control 1:227-236.

15. Deacon, J. W., and Berry, L. A. 1993. Biocontrol of soil-borne plant pathogens: concepts and their application. Pestic. Sci. 37:417-426.

16. Ecke, P., Jr., Matkin, O. A., and Hartley, D. E., eds. 1990. The Poinsettia Manual. Paul Ecke Poinsettias. Encinitas, CA.

17. Escande, A. R., and Echandi, E. 1991. Protection of potato from Rhizoctonia canker with binucleate Rhizoctonia fungi. Plant Pathol. 40:197-202.

18. Gutierrez, W. A., Shew, H. D., and Melton, T. A. 1997. Sources of inoculum and management for Rhizoctonia solani damping-off on tobacco transplants under greenhouse conditions. Plant Dis. 81:604-606.

19. Hagedorn, C., Gould, W. D., Bardinelli, T. R., and Gustavson, D. R. 1987. A selective medium for enumeration and recovery of $P$ seudomonas cepacia biotypes from soil. Appl. Environ. Microbiol. 53:2265-2268. 
20. Henis, Y., Ghafar, A., Baker, R., and Gillespie, S. L. 1978. A new pellet soil sampler and its use for the study of population dynamics of Rhizoctonia solani in soil. Phytopathology 68:371-376.

21. Herr, L. J. 1995. Biological control of Rhizoctonia solani by binucleate Rhizoctonia spp. and hypovirulent $R$. solani agents. Crop Prot. 14:179-186.

22. Homma, Y., Sato, Z., Hirayama, F., Konno, K., Shirahama, H., and Suzui, T. 1989. Production of antibiotics by Pseudomonas cepacia as an agent for biological control of soilborne plant pathogens. Soil Biol. Biochem. 21:723-728.

23. Honeycutt, E. W., and Benson, D. M. 2001. Formulation of binucleate Rhizoctonia spp. and biocontrol of Rhizoctonia solani on impatiens. Plant Dis. In press.

24. Howell, C.R., and Stipanovic, R. D. 1979. Control of Rhizoctonia solani on cotton seedlings with Pseudomonas fluorescens and with an antibiotic produced by the bacterium. Phytopathology 69:480-82.

25. Hwang, J., and Benson, D. M. 2001. Resistance of poinsettia cultivars against Rhizoctonia stem rot induced by binucleate Rhizoctonia. Phytopathology 91:S41.
26. Hwang, J., and Benson, D. M. 2001. Role of pyrrolnitrin produced by Burkholderia $c e$ pacia in biocontrol of Rhizoctonia stem rot of poinsettia. Phytopathology 91:S41.

27. Ichielevich-Auster, M., Sneh, B., Koltin, Y., and Barash, I. 1985. Suppression of dampingoff caused by Rhizoctonia species by a nonpathogenic isolate of $R$. solani. Phytopathology 75:1080-1084.

28. Jayaswal, R. K., Fernandez, M. A., Visintin, L., and Upadhyay, R. S. 1992. Transposon Tn5-259 mutagenesis of Pseudomonas cepacia to isolate mutants deficient in antifungal activity. Can. J. Microbiol. 38:309-312

29. Jones, R. K. 1990. Poinsettia diseases and their management. N. C. Flower Growers' Bull. 35:6-11.

30. Kwok, O. C. H., Gahy, P. C., Hoitink, H. A. J., and Kuter, G. A. 1987. Interactions between bacteria and Trichoderma hamatum in suppression of Rhizoctonia damping-off in bark compost media. Phytopathology 77:12061212.

31. Lemanceau, P., and Alabouvette, C. 1991. Biological control of fusarium diseases by fluorescent Pseudomonas and non-pathogenic Fusarium. Crop Prot. 10:279-286.

32. Loper, J. E., Suslow, T. V., and Schroth, M. N.
1984. Lognormal distribution of bacterial populations in the rhizosphere. Phytopathology 74:1454-1460.

33. Parke, J. L. 1990. Population dynamics of Pseudomonas cepacia in the pea spermosphere in relation to biocontrol of Pythium Phytopathology 80:1307-1311.

34. Poromarto, S. H., Nelson, B. D., and Freeman, T. P. 1998. Association of binucleate Rhizoctonia with soybean and mechanism of biocontrol of Rhizoctonia solani. Phytopathology 88:1056-1067.

35. U.S. Dep. of Agric. 2000. Floriculture crops: 1999 summary. Natl. Agri. Stat. Serv. SpCr 61(00). Washington, D.C.

36. Weller, D. M. 1988. Biological control of soilborne plant pathogens in the rhizosphere with bacteria. Annu. Rev. Phytopathol. 26:379-407.

37. Xue, L., Charest, P. M., and Jabaji-Hare, S. H. 1998. Systemic induction of peroxidases, 1,3 $\beta$-glucanases, chitinases, and resistance in bean plants by binucleate Rhizoctonia species. Phytopathology 88:359-365.

38. Yuen, G. Y., Craig, M. L., and Giesler, L. J. 1994. Biological control of Rhizoctonia solani on tall fescue using fungal antagonists. Plant Dis. 78:118-123. 